non-spherical particles, and, in reply, Dr. Rose pointed out that some of the theoretical conclusions are supported by published results relating to the milling of chert and dolomite, both of which are non-spherical and polydisperse, and so, at least as a first approximation, the theory appears to be applicable to such materials.

Mr. R. L. Brown showed that, by the use of matrix algebra, the performance characteristics of a closedcircuit milling installation may be deduced from the relatively simple performance characteristics of the elements of the system. Such methods, however, demand an accurate knowledge of the performance characteristics of the elements, and in this connexion accurate laws of comminution are desirable. The existence of an inter-relationship between the various elements of a mill installation at once raises the question of the extent to which the 'laws' found by experiment are dependent upon the nature of the material undergoing treatment and upon the charac. teristics of the plant involved.

\section{REACTIONS AND STRUCTURE IN CARBOHYDRATES}

\begin{abstract}
A SYMPOSIUM on "Newer Interpretations of Reactions and Structure in Carbohydrate Chemistry" was held under the auspices of the Chemical Society on November 1, in the Chemistry Department of University College, London. 'There was a large and interested audience composed mostly of the younger chemists. It was a particular pleasure to note the presence of Prof. C. K. Ingold, hast tin the meeting, and of Prof. E. L. Hirst, president of the Chemical Society.
\end{abstract}

Dr. G. R. Barker, the organizer of the symposium, was in the chair for the afternoon session, when the opening speaker was Prof. E. J. Bourne (Royal Holloway College, London), who discussed some physical methods used in the determination of carbohydrate structures. He first outlined the contributions which infra-red analysis recently carried out in the University of Birmingham can make to the solution of many problems encountered in carbohydrate chemistry. Among the applications are the direct comparisons of unknown samples with authentic specimens, analyses of mixtures, determinations of hydrogen bonding and degrees of crystallinity, detection of substituents and of water of crystallization, etc. In suitable cases, infra-red techniques provide information on configuration, conformation, ring size, anomeric state and positions of glycosidic linkages in polysaccharides. The assignment of the more important vibrations was considered. Preliminary results in studies of the Raman spectra of ring systems in carbohydrates and their derivatives were presented. Reference was made to Dr. D. H. Whiffen's theoretical interpretation of optical activity in carbohydrates and to its possible applications in future work.

Dr. J. C. P. Schwarz (University of Edinburgh) then spoke on conformational ideas applied to tosyl esters and three-membered oxide rings. In certain carbohydrate derivatives, equatorial hydroxyl groups are tosylated more readily than axial hydroxyl groups. Replacement reactions of primary sulphonyloxy groups (for example, tosyl esters) with sodium iodide, sodium hydroxide, lithium aluminium hydride, and other reagents probably proceed by $S_{N} 2$ mechanisms with carbon-oxygen fission. In the absence of participation of neighbouring groups, carbohydrate derivatives containing secondary sulphonyloxy groups attached directly to a ring are generally much less reactive than primary sulphonyloxy compounds, and sulphur-oxygen fission is usually involved in their reactions with sodium hydroxide or lithium aluminium hydride.

'The relative reactivities of various tosyloxy compounds are influenced by two types of steric effect, namely, hindrance encountered by the incoming group and changes occurring in the non-bonded repulsions between substituents as the molecule passes from its initial state to the transition state. The first type is illustrated by the behaviour of the $2: 5$-di-O-tosyl-1 :4-3:6-dianhydro hexitols, and the second is important in elimination and cyclization reactions which involve groups attached to a neighbouring carbon atom. The migration of three. membered oxide rings was discussed at some length.

Dr. A. B. Foster (University of Birmingham) then followed with a paper on conformational aspects of the formation and reactions of certain cyclic derivatives of carbohydrates. A number of reactions in carbohydrate chemistry are amenable to conformational analysis. Those particularly discussed were: (i) preferential formation of certain cyclic acetals in the reactions of aldehydes and polyalcohols in the presence of acid; (ii) the position of the equilibrium established in aqueous acid between a hexose and its $1: 6$-anhydro-pyranoside derivative; (iii) the remarkably facile isomerization undergone in the presence of aeid by derivatives of certain $3: 6$-anhydrohexopyranosides; and (iv) a number of novel steric effects which have been encountered in the study of the reaction of borate ions with pyranoside derivatives. Some of these were explained by postulating the participation of boat forms of the carbohydrate in the reaction with borate ions. The discussions of the two papers were taken together. The speakers, particularly Dr. Schwarz, were bombarded with questions, and conformational analysis was evoked to explain various other reactions, including some biochemical changes.

The chairman for the evening session was Prof. M. Stacey. Dr. W. G. Overend (Birkbeck College, London) gave a valuable paper on the hydrolysis of glycosides. The acidic hydrolysis of $\mathrm{O}$-glycosides and glycosylamines was discussed from the point of view of various factors such as the variation of the glycosidic substituent; configurational change at the glycosidic centre; variation of substituents on the glycopyranosyl residue and alteration in the size of the sugar ring. Conformational and mechanistic aspects of glycoside hydrolysis were outlined, and consideration given to the formation and hydrolysis of glycosyl phosphates and to the transglycosylation reaction.

The final comprehensive paper was given by a distinguished guest from Ottawa, Dr. R. U. Lemieux (University of Ottawa), who discussed reactions at the anomeric centre of acetylated glycopyranosyl acetates and halides. The reactions appear almost invariably to involve participation of either the ring. oxygen atom or the $C(2)$-substituent. The participations strongly affect the stereochemical route of reaction and appear to impose the half-chair conformation on the pyranose ring of the intermediate state. A consideration of the interactions between non-bonded atoms in the half-chair conformation of 
the intermediate state appears to be more fruitful in the rationalization of observed effects of configuration on reactivity than are similar considerations based on the conformation of the reactant in the ground state.

The use of proton magnetic resonance spectroscopy for establishing the conformations of sugar acetates was briefly discussed.

The symposium altogether was a lively and stimulating affair, and in the closing remarks the chairman commented on the increasing importance of applications of these newer interpretations of carbohydrate reactions in the ever-widening field of biochemistry.

\section{ASSOCIATION OF SCHOOL NATURAL HISTORY SOCIETIES \\ EIGHTH ANNUAL EXHIBITION, 1956}

$\mathrm{T}$

HE eighth annual exhibition of the Association of School Natural History Societies was held at the British Museum (Natural History) on October 20. Mr. Eric Hosking, who is a founder-member of the Association, gave a lecture on "Birds in Action" before an audience of 150 children. This was illustrated by a selection of magnificent photographs of birds, for which Mr. Hosking is rightly famous. By means of electronic flash and occasionally with the uso of a specially designed photo-electric camera release, some remarkable pictures had been obtained.

After the lecture, the exhibition was opened to members and to the public. A notable feature of this year's exhibition was the reduction in the number of charts and diagrams coupled with an increase in live exhibits. While it is true that the tabulation of results and their presentation in graphic form is an admirable, if not essential, way of summarizing field work, it is also true that this practice often makes a dull exhibit. Nine out of the twelve items displayed by Bishop's Stortford College were living exhibits, including an elaborate reconstruction of a portion of a refuse dump. In another exhibit were specimens of the white-lipped hedge snail (Cepea nemoralis), showing the many variants of shell-marking. African clawed frogs, reared at the school, made an interesting contrast to familiar British amphibians. Other examples of living freshwater animals were shown by Oundle School, Felsted School and Lord Wandsworth College, Hants. The latter school displayed also a home-made portable instrument for the field measurement of salinity in salt marshes. This operated by measuring the electrical conductivity of the water contained in a long polythene tube. The study of the natural history of mammals is a subject which has been sadly neglected by the majority of naturalists. It was therefore encouraging to see that Kimbolton School, Hunts, had begun a scheme of trapping and marking small mammals. A number of living small rodents was displayed.

Marine studies had been undertaken by Dulwich College, which displayed a large collection of marine algae and a shore transect illustrating the zonation of these species on the sea-shore. Similar work was described by Ackworth School, Yorkshire.

A pupil of Bishop's Stortford College had prepared a model to illustrate the trapping of moths at a mercury-vapour light trap. A relief model of an area of countryside, above which bird flight-lines were indicated, showed that whereas some species of bird preferred to fly along the valleys, other species tended to fly in a direction perpendicular to the contour lines. Limehurst Natural History Society (Garendon School, Loughborough, Leics.) displayed models, photographs and charts which described their island nature reserve and the wild life found there.

Lepidoptera were not so prominent in the exhibition as they were last year. Several schools showed a few in connexion with their other exhibits, but the collection shown by Tonbridge School was the most comprehensive.

Geology was represented by a soil profile across an area of poor fen, exhibited by Cranbrook School, and a selection of rock samples and fossils from an ironstone cutting brought by Uppingham School. Also included in their exhibits were owl pellets and kestrel pellets, whole and dissected. Owl pellets were featured in the displays by Oundle School, and the William Grimshaw School, London. Tho latter school had prepared a complete demonstration of the various stages in the dissection of a pellet, culminating in the 'reconstruction' of most of the skeleton of an individual vole from bones found in a single pellet.

It was announced that further exhibitions have been arranged in Leicester (November 24) and Leeds (May 11, 1957). Other meetings are being planned to take place in Exeter and Manchester. Further details of these meetings can be obtained from $\mathrm{O}$. N. Bishop, Hon. General Secretary, A.S.N.H.S., 115 Shelly Road, Exmouth, Devon. A special edition of the Exhibition Catalogue is available $(10 \mathrm{~d}$, including postage), which contains a full list of exhibits, a list of members and their addresses, and the annual reports of the committee.

In addition to those displays put on by member schools there were a number of guest exhibitors. The Amateur Entomologists' Society, the Royal Society for the Protection of Birds, the School Nature Study Union and the Universities Federation for Animal Welfare displayed their publications. The Children's Centre showed material illustrating their Saturday morning activities; a scale-model of a, small portion of Epping Forest was particularly noteworthy.

O. N. BISHOP

\section{EXPEDITION TO THE SALMON AND LEDUC GLACIERS}

$\mathrm{A}$

$\mathrm{N}$ expodition to the Salmon and Leduc Cxlaciers A was organized in 1956 by the University of Toronto under the leadership of Prof. J. A. Jacobs at the request of the Defence Research Board and the National Research Council of Canada. Eleven men took part, and the expedition lasted from tho middle of May until the end of August.

The Salmon and Leduc Glaciers, which are situated near the coast of British Columbia opposite the southern tip of Alaska (approximately lat. $56^{\circ} 10^{\prime}$ N., long. $130^{\circ} 15^{\prime} \mathrm{W}$.), were chosen since they lie betwoen the glaciers from the Juneau ice field to the north and those of the Washington Cascado and Olympic Mountains to the south, on which a considerable amount of work has already been done. The area is also interesting since the Granduc ore body lies in the mountains surrounded by these and other glaciers. 